\title{
Peutz-Jegher's syndrome presenting as jejunoileal intussusception in an adult male: a case report
}

\author{
Hardik H Thakker ${ }^{1}$, Amita Joshi ${ }^{2}$ and Aparna Deshpande ${ }^{3}$
}

Addresses: ${ }^{1}$ Seth G S Medical College and KEM hospital, Parel, Mumbai 400012, India

${ }^{2}$ Department of Pathology, Seth G S Medical College and KEM hospital, Parel, Mumbai 400012, India

${ }^{3}$ Department of General Surgery, Seth G S Medical College and KEM hospital, Parel, Mumbai 400012, India

Email: HHT* - hardikthakker@hotmail.com; AJ - drjoshiamita@yahoo.co.in; AD - draparnadeshpande@yahoo.com

* Corresponding author

Received: 16 October 2008 Accepted: 23 February 2009 Published: II August 2009

Cases Journal 2009, 2:8865 doi: 10.4076/1757-1626-2-8865

This article is available from: http://casesjournal.com/casesjournal/article/view/8865

(C) 2009 Thakker et al.; licensee Cases Network Ltd.

This is an Open Access article distributed under the terms of the Creative Commons Attribution License (http://creativecommons.org/licenses/by/3.0), which permits unrestricted use, distribution, and reproduction in any medium, provided the original work is properly cited.

\begin{abstract}
Introduction: Peutz-Jegher's syndrome is a rare autosomal dominant disorder that typically manifests itself as recurrent colicky abdominal pain and blood loss in stools. In adults, it is only rarely accompanied by frank intussusception and intestinal obstruction. We encountered an adult Asian Indian male who presented with an intestinal obstruction due to jejunoileal intussusception. It was caused by a $3.5 \mathrm{~cm}$ large hamartomatous polyp of Peutz-Jegher's syndrome. We feel reporting the unusual presentation of this rare condition may be a noteworthy contribution to the scarce literature on Peutz-Jegher's syndrome from India. The case report may be of educational importance to the clinicians and students because it is unusual to see this case in typical clinical practice.

Case presentation: A 38-year-old Asian Indian male presented to us in the surgical emergency room with colicky abdominal pain, reporting vomiting and blood in stools over the previous two days. Clinical examination suggested intestinal obstruction. Ultrasonography of the abdomen showed signs of intussusceptions, which were then confirmed by an emergency exploratory laparotomy. We resected the intussuscepted small bowel segment and performed a jejuno-ileal anastomosis. A histopathology examination of the resected specimen revealed multiple hamartomatous polyps suggestive of Peutz-Jegher's syndrome. In this case report, we present the pathology findings, their clinical correlation and a detailed discussion of Peutz-Jegher's syndrome and adult intussusception. We also discuss its other rare presentations reported in literature.
\end{abstract}

Conclusion: Hamartomatous polyps of Peutz-Jegher's syndrome can sometimes grow to a large size and form the lead point of an intussusception.

\section{Introduction}

Peutz-Jegher's syndrome is a rare autosomal dominant disorder with variable penetrance. It is characterized by mucocutaneous hyperpigmentation, hamartomatous polyps of the gastrointestinal tract and multiple neoplasms.
It commonly presents as recurrent colicky abdominal pain and blood loss in stools. An adult Asian Indian patient presented to us with an intestinal obstruction. This was secondary to a large hamartomatous jejunal polyp causing intussusception. Only $5 \%$ to $15 \%[1,2,3]$ of 
intussusceptions occur in adults and they contribute to only $1 \%[4,5]$ of all causes of intestinal obstruction. A report of this unusual presentation of a rare condition may be a noteworthy contribution to the scarce literature on PeutzJegher's syndrome from India.

\section{Case presentation}

A 38-year-Asian Indian male presented to us in the emergency room with colicky abdominal pain in left lower and umbilical area, vomiting and blood in stools over the previous 2 days. He did not have a history of fever. He had a history of intermittent abdominal pain over the previous 2 months. His family history was not significant. On examination he was afebrile, with a pulse of $92 / \mathrm{min}$ regular and blood pressure of 130/88 mm Hg. The lower labial and buccal mucosa showed hyper pigmentation bilaterally (Figure 1). There was tenderness and guarding in the left iliac fossa. There
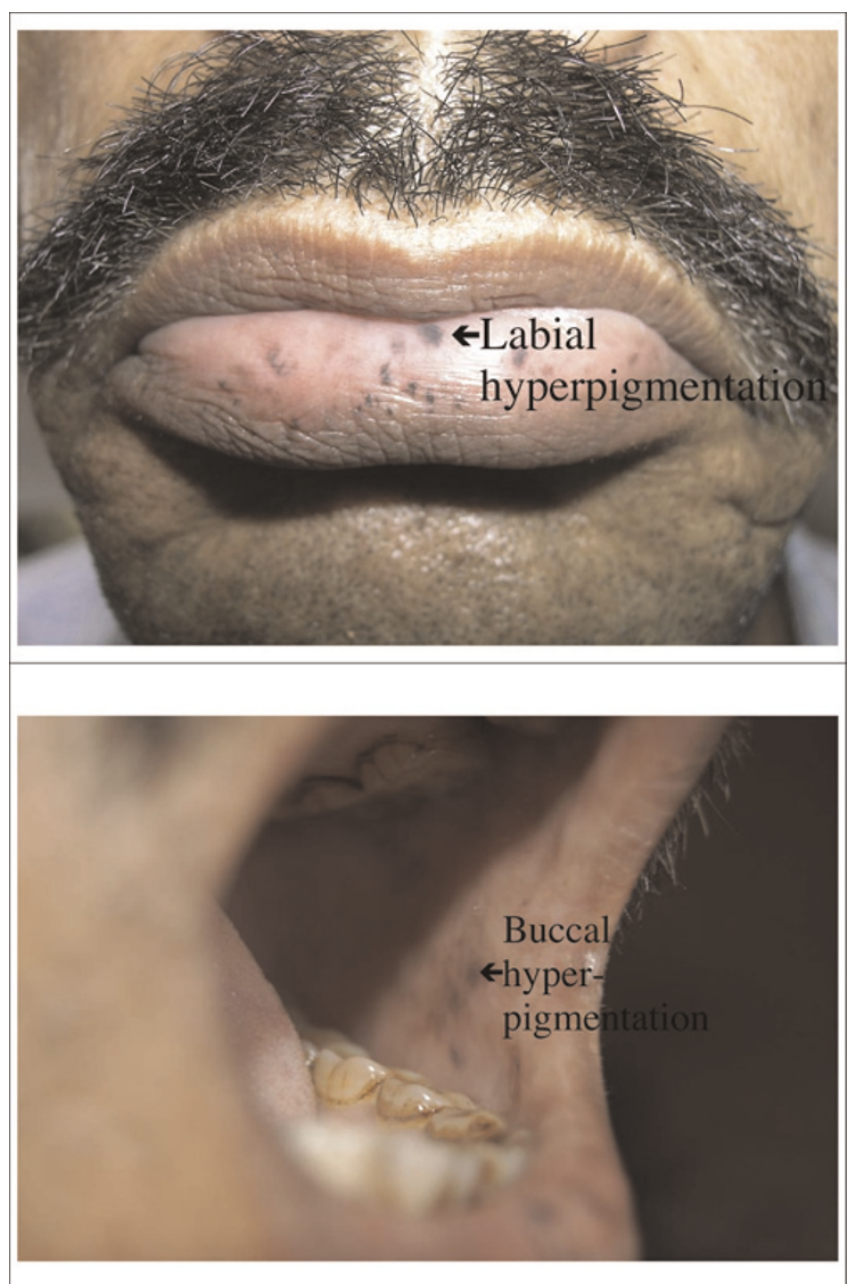

Figure I. Shows clinical photographs of the patient with labial and buccal hyperpigmentation. was no lump, organomegaly or free fluid. Hernial orifices were normal. However, bowel sounds were increased.

Blood investigations showed severe anemia ( $\mathrm{Hb}: 7 \mathrm{gm} / \mathrm{dl}$ ). A plain x-ray of the abdomen was unremarkable. Abdominal ultrasonography showed two echo complex areas with concentric rings and target appearance: one in the left lumbar region and the other one of size $3.5 \mathrm{~cm} \times 5 \mathrm{~cm}$ in the hypochondriac. This was suggestive of an intussusception.

We started the patient on intravenous fluids and antibiotics. We inserted a nasogastric tube and transfused him with two units of whole blood. We then performed an emergency exploratory laparotomy. Intra-operatively we found a one-foot-long segment of jejuno-ileal intussusception, extending approximately three feet distally from the duodenojejunal flexure. We resected the intussuscepted segment and performed a jejuno-ileal anastomosis. We found multiple polyps on the jejunal resection margins. We sent the resected segment for histopathology examination. We then placed a drain and closed the abdomen. His recovery in the wards was uneventful. We discharged him 8 days after the surgery.

\section{Gross pathology findings}

The specimen of small intestine was $32 \mathrm{~cm}$ in length, with grey serosa and unremarkable cut section. There was no evidence of gangrenous changes. The mucosa was lined by multiple polyps that were scattered and on average measuring approximately $1 \mathrm{~cm}$ in diameter. A single large pedunculated polyp measuring $3.5 \mathrm{~cm}$ in diameter was attached to the mucosa with a stalk $1.1 \mathrm{~cm}$ in length, located $2 \mathrm{~cm}$ from the proximal resected margins. The polyps were reddish grey in color (Figure 2).

\section{Histopathology}

Multiple sections studied from the polyps showed features of hamartomatous polyps, composed of glands separated by a band of smooth muscles (Figure 2). There was no evidence of atypia or malignancy. Lymph nodes showed reactive hyperplasia.

\section{Diagnosis}

Peutz-Jegher's Syndrome. We subjected him to regular follow up and a strict surveillance for other neoplasms common in Peutz-Jegher's syndrome. We could not carry out a mutation analysis for STK 11/LKB 1 gene due to lack of resources.

\section{Discussion}

The occurrence of adult intussusception is rare $[1,2,3]$. Its characteristics in adults are different from its characteristics in children in some notable respects as indicated in Table 1. Peutz-Jegher's syndrome is a rare autosomal disorder with variable penetrance characterized by mucocutaneous hyper pigmentation, hamartomatous polyps of 


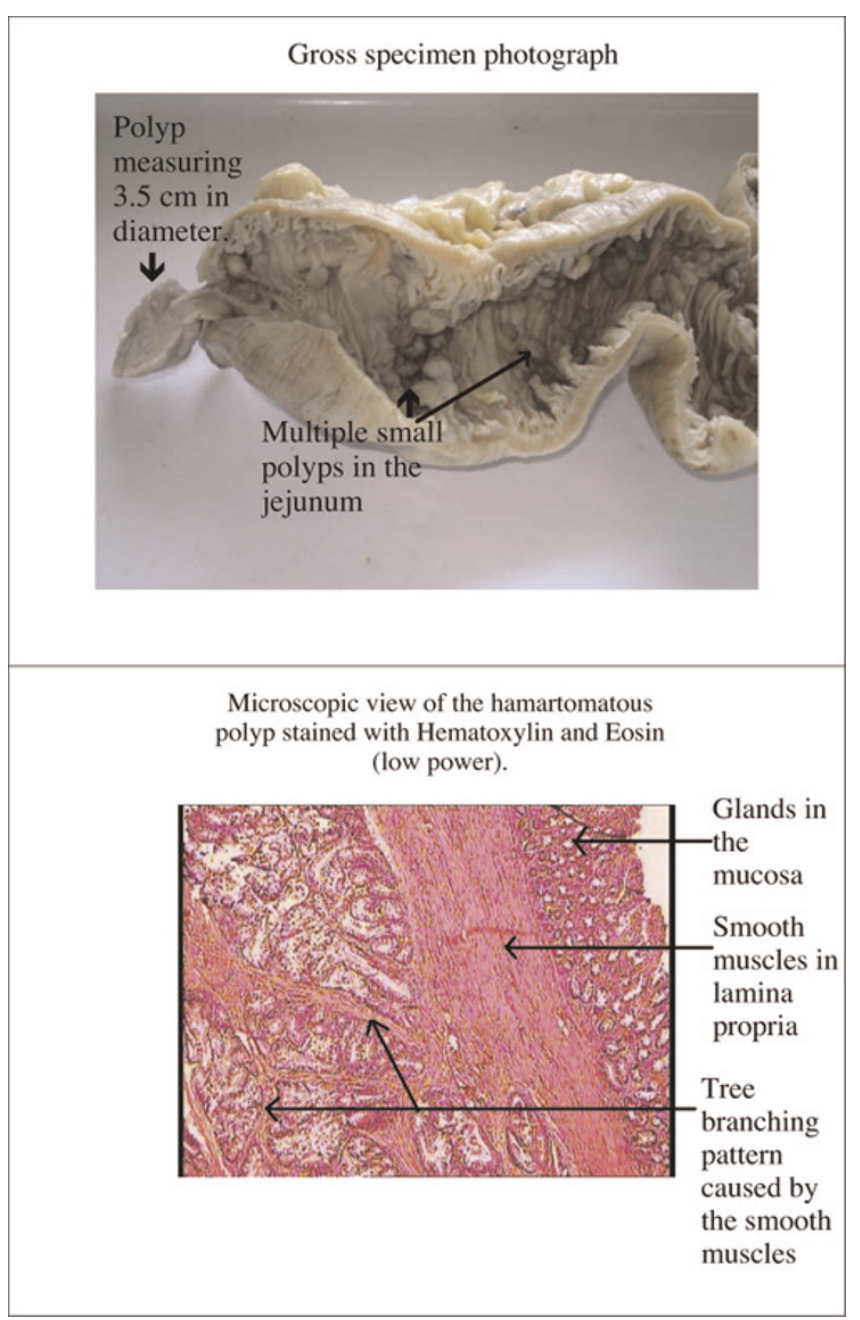

Figure 2. Shows a gross pathology photograph of the resected segment of the small bowel with a large hamartomatous polyp. This polyp formed the lead point for intussusception. It also shows a low power microscopic view of a hamartomatous polyp stained with Hematoxylin and Eosin.

gastrointestinal tract and multiple neoplasms. The diagnostic criteria for the syndrome are as follows: a) Three or more histologically confirmed Peutz-Jegher's polyps or b) Any number of Peutz-Jegher's polyps with a family history or c) Characteristic prominent mucocutaneous pigmentation with a family history or d) Any number of Peutz-Jegher's polyps with characteristic prominent mucocutaneous pigmentation. It commonly presents as recurrent colicky pain in abdomen and blood loss in stools. The polyps in this condition are hamartomatous and rarely undergo malignant transition but a few such cases have been reported in literature [6]. The patients are prone to many extra intestinal tumors like testicular sertoli tumors, ovarian tumors like sex cord tumors with annular tubules, granulose theca cell tumors, cystadenomas, breast tumors like breast carcinoma, papilloma with squamous metaplasia, cholangioma, pancreatic adenocarcinoma, adenoma malignum, bronchial carcinoids, papillomas in bladder and pelvis [7]. Patients observed with such conditions need to be placed under regular surveillance to facilitate early detection and regular followup.

Patients may also have other non neoplastic extra intestinal lesions like nasal polyps, gall bladder polyps, nodular goiter and adrenal cortical hyperplasia [7].

Recently, gene mutation studies have proved that mutation in Serine Threonine kinase gene STK 11/LKB 1 located on 19p.13.3 [8], is the cause of Peutz-Jegher's. However, the exact mechanism by which it affects protein synthesis and causes polyp formation is yet to be elucidated. Cases of intussusception in Peutz-Jegher's have been reported in children $[9,10,11]$ but have been rare in adults. Cases of Peutz-Jegher's with carcinoid [12], duodenal [13] and gastric [14] carcinomas have also been reported.

\section{Correlation of pathologic findings}

The largest polyp measuring $3.5 \mathrm{~cm}$ in size was the lead point causing telescoping of jejunum into ileum, thereby causing abdominal pain, guarding and currant jelly stools. The anemia could be attributed to bleeding from the multiple hamartomatous polyps, but we could not rule out other causes like ulcer disease, and iron deficiency.

\section{Future advances}

Once the exact mechanism by which mutations in STK 11/LKB1 cause Peutz-Jegher's Syndrome is known, it will open doors of advance treatment modalities like gene therapy.

Table I. The characteristics of intussusception in adults and children

\begin{tabular}{lll}
\hline & Adult intussusception & Intussusception in children \\
\hline Frequency & $5 \%$ of all cases & $95 \%$ of all cases \\
Part of the bowel involved & Commonly involves small bowel & Usually involved colon \\
Symptoms & Classical symptoms of intussusception are not always & Classical symptoms are present in many cases \\
& present & Idiopathic due to lymphoid hyperplasia in Peyer's patches \\
Cause & Caused by malignancy (50\%) or polyp & Treatment is hydrostatic reduction and if needed manual \\
Treatment & Treatment is usually resection of intussuscepted & reduction by laparotomy, resection not needed unless \\
& segment without reduction (to prevent seedling of & gangrene sets in \\
\hline
\end{tabular}




\section{Conclusion}

Hamartomatous polyps of Peutz-Jegher's syndrome can sometimes grow to a large size and form the lead point of an intussusception.

\section{Abbreviation}

$\mathrm{Hb}$, haemoglobin.

\section{Consent}

Written informed consent was obtained from the patient for publication of this case report and accompanying images. A copy of written consent is available for review by the editor-in-chief of this journal.

\section{Competing interests}

The authors declare that they have no competing interests.

\section{Authors' contributions}

HHT carried out a review of the literature, investigated the patient case records, obtained clinical, gross pathology and histopathology pictures, drafted and obtained informed consent from the patient, obtained no objection letter from the Ethics Committee, drafted the manuscript. AD performed an initial evaluation of patient, operated on him, helped in the review of the literature, contributed to the clinical aspects of the case and editing of the manuscript. AJ performed gross and histopathology examination of the resected gut specimen, contributed to the pathology findings of the report and helped in the review of the literature and editing of the manuscript. All authors read and approved the final manuscript.

\section{Acknowledgements}

We wish to thank the patient for his cooperation and consent for permitting us to use his medical records and clinical photographs for this case report. (A written informed consent was obtained for the purpose).

We also thank the Dean of Seth G S Medical College and KEM Hospital, and associated staff for permitting us to use the medical records of the patient for this case report and to publish it. A special thanks to the Ethics Committee of the hospital for their co-operation. (A no objection letter was obtained from the Ethics Committee of KEM Hospital for submitting the case report).

\section{References}

I. Laws HL, Alderate JS: Small bowel obstruction a review of $\mathbf{4 6 5}$ cases. South Med J 1976, 69:733-734.

2. Stewardson RD, Bombect CT, Nyhers LM: Critical operative management of small bowel obstruction. Ann Surg 1978, 187:189-193.

3. Briggs DF, Arpathios J, Zollinger RW: Intussusception in adults. Am J Surg 1961, 101:109-113.

4. Domhauser JL, Kelly EC: Intussusception in adults. Am J Surg 1950, 79:673-677.

5. Agha FP: Intussusception in adults. AJR Am J Roentgenol 1986, |46:527-53I. Roentgenology.
6. Mehta MV, Porecha MM, Mehta PJ: Small intestinal adenocarcinoma in Peutz-Jeghers syndrome. Indian J Gastroenterol 2006, 25:38-39.

7. Cecilia M, Fenoglio-Preiser: Peutz Jegher's syndrome. In Gastrointestinal Pathology: an atlas and text. 3rd edition. Philadelphia: Lippincott Williams and Wilkin; 2008:704-7II.

8. Hemminki A, Tomlinson I, Markie D, Jarvinen $H$, Sistonen $P$, Bjorkqvist AM, Knuutila S, Salovaara R, Bodmer W, Shibata D, de la Chapelle A, Aaltonen LA: Localization of a susceptibility locus for Peutz-Jeghers syndrome to $19 p$ using comparative genomic hybridization and targeted linkage analysis. Nat Genet 1997, 15:87-90.

9. Trifonov G, Ivanchev I, Apostolova A, Khristov KH: A case report of Peutz-Jeghers syndrome in childhood. Khirurgiia (Sofiia) I99।, 44:16-19.

10. Mendes da Costa P, Reygaerts D, Desorgher G, Vandendael P, Rutsaert ], de Temmerman P: [Peutz-Jeghers syndrome. Description of a case and literature review]. Acta Gastroenterol Belg 1009 , 53:180-187.

II. Sokmen HM, Ince AT, Bolukbas C, Kilic G, Dalay R, Kurdas OO: A Peutz-Jeghers syndrome case with iron deficiency anemia and jejuno-jejunal invagination. Turk J Gastroenterol 2003, 14:78-82.

12. Wada K, Asoh T, Imamura T, Tanaka N, Yamaguchi K, Tanaka M: Rectal carcinoid associated with Peutz Jeghers syndrome. J Gastroenterol 1998, 33:743-746.

13. Lin JI, Caracta PF, Lidner A, Gutzman LG: Peutz-Jeghers polyposis with metastasizing duodenal carcinoma. South Med J 1977, 70:882-884.

14. Cochet B, Carrel J, Desbaillets L, Widgren S: Peutz-Jeghers syndrome associated with gastrointestinal carcinoma: report of two cases in a family. Gut 1979, 20:169-175.

\section{Do you have a case to share?}

\section{Submit your case report today}

- Rapid peer review

- Fast publication

- PubMed indexing

- Inclusion in Cases Database

\section{Any patient, any case, can teach us something}

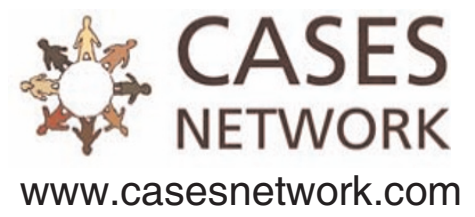

